\title{
Artrópodos associados à copa de Attalea phalerata Mart. (Arecaceae), na região do Pantanal de Poconé, Mato Grosso, Brasil
}

\author{
Geane Brizzola dos Santos ${ }^{1}$ \\ Marinêz Isaac Marques ${ }^{2}$ \\ Joachim Adis ${ }^{3}$ \\ Carlo Ralph De Musis ${ }^{4}$
}

\begin{abstract}
Arthropods associated with the canopy of the palm Attalea phalerata Mart. (Arecaceae), in the Pantanal of Poconé, Mato Grosso, Brazil. Six individuals of the palm A. phalerata, in Poconé floodplains of Mato Grosso, were sprayed with a synthetic pyrethroid $(0.25 \%$ concentration $)$ in order to study the biomass, diversity, and richness of the canopy arthropods. A total of $17,188\left(238.7 \pm 80.6\right.$ ind.$\left./ \mathrm{m}^{2}\right)$ arthropods belonging to 22 Orders, was collected in a $72 \mathrm{~m}^{2}$ funnel area. Two hours after spraying, $58.9 \%$ of the total number fell into the funnels, $37.6 \%$ was obtained by shaking the trees, and finally, $3.5 \%$ after cutting and washing all the palm leaves. The Coleoptera (27.4\%), Hymenoptera-Formicidae (19.0\%), Collembola (13.6\%), Psocoptera (10.7\%), Diptera $(9.0 \%)$ and Araneae $(6.4 \%)$ were the predominant. The total biomass was $15.1 \mathrm{~g}$ dry weight $\left(0.4 \mathrm{mg} / \mathrm{m}^{2} ; 0.13+0.04 /\right.$ tree $)$. A total of 4,715 beetles representing 48 families and 326 morphospecies were obtained. Tenebrionidae $(22.9 \%)$, Curculionidae $(22.0 \%)$, Carabidae $(10.9 \%)$ and Staphylinidae $(7.9 \%)$ were the most abundant, while Curculionidae (44 spp.), Staphylinidae (40 spp.) and Chrysomelidae (34 spp.) presented the largest number of morphospecies. Herbivores $(37.5 \%)$ were the dominant in the trophic guilds of adult Coleoptera, followed by predators (35.4\%), fungivores (14.6\%), and saprophages (12.5\%). Although most arthropod Orders were represented in all the palms sampled, analysis of variance showed no significant differences in their composition, however there was a significant difference in their frequency of occurrence.
\end{abstract}

KEYwords. Arthropods; Attalea; biomass; guilds; Pantanal.

\section{INTRODUÇÃO}

As copas de árvores abrigam ricas comunidades de artrópodos por apresentarem complexa estrutura que fornece uma grande diversidade de nichos (LAWTON 1978, 1982), recursos estáveis (Southwood 1961, 1978), aparência que estimula potenciais colonizadores (FEENY 1975, 1976), além das espécies da comunidade componente estarem troficamente interligadas (Moran \& SouthwOOD 1982).

Os estudos relacionados com artrópodos arbóreos intensificaram-se com o desenvolvimento de novas técnicas de acesso às copas (STORK et al. 1997; LinSENMAIR et al. 2001; BASSET et al. 2003). Desde então, essas comunidades têm sido investigadas com o objetivo de conhecer sua composição taxonômica, padrões alimentares, guildas tróficas, distribuição, abundância e diversidade.

As investigações com fauna de artrópodos associada às copas de árvores tropicais restringem-se às florestas tropicais úmidas e secas. No Brasil, estas pesquisas concentraram-se na região amazônica, comparando áreas inundáveis e de terra firme (Adis et al.1984, 1998a; Erwin 1983a; HuRTADO GuerRero et al. 2003; HARADA \& ADIS 1998).

No Pantanal de Mato Grosso alguns aspectos como estrutura de guilda de insetos, padrões de herbivoria e diversidade genética em Tabebuia aurea (Manso) Benth. \& Hook. e T. ochraceae (Cham.) Standl. (Bignoniaceae), foram abordados por RiBEIRO \& BROWN (1999). MARQUES et al. (2001) estudaram a composição taxonômica de artrópodos em copa

1. Departamento de Zoologia, Universidade Federal do Paraná. Caixa Postal 19030, 81531-980 Curitiba-PR, Brasil.

2. Departamento de Biologia e Zoologia, Universidade Federal de Mato Grosso. Av. Fernando Correa da Costa, s/n, Coxipó, 78060-900 CuiabáMT, Brasil.

3. Max-Planck-Institute for Limnology, Tropical Ecology Working Group. Postfach, 165, D-24302 Plön, Alemanha

4. Faculdade de Educação, Pontifícia Universidade Católica. Rua Monte Alegre, 984, 05014-901 Perdizes-SP, Brasil. 
de Vochysia divergens Pohl. (Vochysiaceae), uma espécie invasora de campos no Pantanal de Mato Grosso.

As pesquisas com a fauna de artrópodos sobre copas de palmeiras ainda são escassas, sendo que a maioria limita-se a espécies-praga (JANZEN 1971; LiNK \& AlVAREZ 1979; NiLSSON \& Johnson 1993; Johnson et al. 1995; Delgado 2002), polinizadores (SCHMidT 1970; Mora UrPi \& Solis 1980; Mora UrPi 1982; Wright 1990; Scariot et al.1991; Kahn \& De GranVILLE 1995; MACAMBIRA \& JARDIM 1997; KüChMEISTER et al. 1998; Listabarth 1999; Dulmen 2001; Quiroga-Castro \& ROLDÁN 2001) e outros tratam apenas de grupos de insetos (VAsConcelos 1990; Costa et al.1992).

As palmeiras fazem parte de ecossistemas naturais, fornecendo alimento e abrigo para diversos grupos de animais. As flores e frutos são utilizados como recursos para a sobrevivência de artrópodos, aves, roedores e outros animais (Wilson \& Janzen 1972; Wrigh 1983; Kahn \& De Granville 1995; Flechtmann 1997; Adler \& Kestell 1998; Jardim \& DA CunHa 1998; Harms \& Dalling 2000).

As palmeiras arborescentes, na maioria, são plantas altamente produtivas e, em florestas inundáveis, são dominantes em alguns ambientes, desempenhando importante papel como fonte de matéria orgânica, devido à persistência e fibrosidade das bainhas e à presença de folhas mortas sobre o tronco (Kahn \& De Granville 1995).

As espécies da família Arecaceae adaptam-se a diversos tipos de florestas como terra firme, campinarana, periodicamente inundadas, e em diversos ambientes degradados (MirANDA et al. 2001).

No Pantanal mato-grossense, Attalea phalerata (Arecaceae) ocorre em solos aluviais periodicamente inundáveis (HENDERSON et al. 1995). É também encontrada em capões consorciada com outras espécies vegetais, podendo ocorrer como monodominante em adensamentos conhecidos como acurizais (NUNES DA CUNHA \& JUNK 1999).

Nunes da Cunha \& Junk (1999, 2001), em um estudo da composição florística na região do Pantanal de Poconé, Mato Grosso, Brasil, registraram a ocorrência desta espécie nos gradientes de localização denominados como áreas inundáveis em cheias excepcionais e área com curto tempo de inundação.

Considerando a importância de $A$. phalerata como unidade de paisagem pantaneira, por ocorrer como espécie monodominante, e o desconhecimento de sua artropodofauna, esta pesquisa objetivou amostrar a comunidade de artrópodos associados à sua copa, por meio da utilização de pulverização com inseticida.

\section{MATERIALE MÉTODOS}

O experimento foi realizado em um acurizal localizado na Fazenda Retiro Novo, distrito Pirizal, município de Nossa Senhora do Livramento, situada à margem direita do rio Cuiabá e margem esquerda do rio Bento Gomes ( $16^{\circ} 15^{\prime} 12^{\prime}$ 'S, 56 22' 12 ' W). Esta região está inserida na unidade Planícies e Pantanais Mato-grossenses como Pantanal de Cuiabá-Bento-GomesParaguaizinho, denominado Pantanal de Poconé, e situa-se a sudoeste do Estado de Mato Grosso, Brasil, entre as coordenadas geográficas de $16^{\circ} 15^{\prime} 24^{\prime \prime}$ e $17^{\circ} 54^{\prime} 32^{\prime}$ " de latitude sul e $56^{\circ} 36^{\prime} 24^{\prime \prime}$ e $57^{\circ} 56^{\prime} 23^{\prime \prime}$ de longitude oeste (FrANCO \& PiNHEIRO 1982).

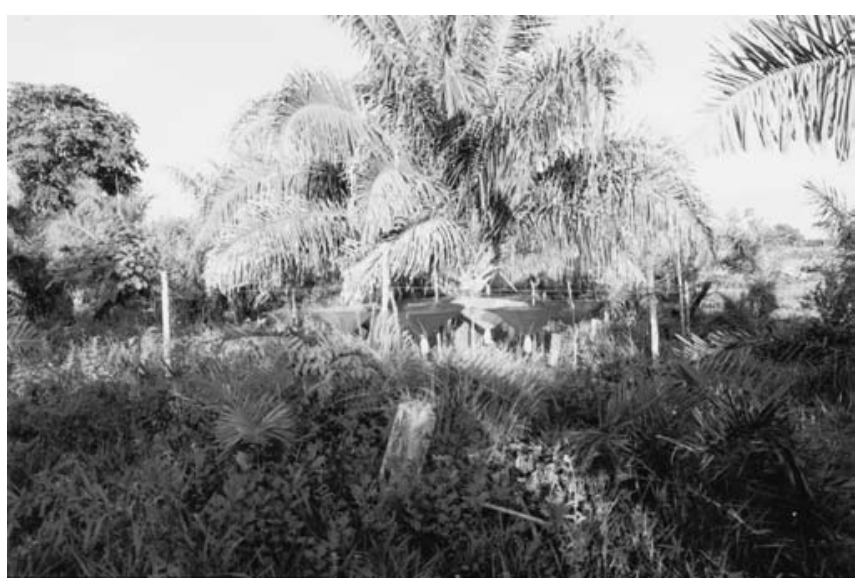

Fig. 1. Disposição dos funis abaixo da copa de Attalea phalerata Mart. (Arecaceae) para a coleta da artropodofauna.

Seis árvores de $A$. phalerata foram amostradas utilizandose o método de pulverização com inseticida, nos meses de novembro e dezembro de 1999. Estes meses correspondem ao início das chuvas (ADÁMOLI 1986; TARIFA 1986; CAMPELO JR. et al.1997; NunES DA CUNHA \& JUNK 1999) mas, excepcionalmente neste ano, ainda mantinham características do período de seca. Para a seleção das árvores e coleta dos artrópodos seguiramse os critérios indicados por AdIs et al. (1998b).

Nas seis árvores amostradas foram tomadas medidas do diâmetro do fuste na altura do peito (DAP), altura total e diâmetro da copa (Tabela I). Para a coleta dos artrópodos, 12 funis de nylon de $1 \mathrm{~m}$ de diâmetro foram instalados abaixo da copa de cada árvore. Estes funis, suspensos por cordas amarradas em estacas e no próprio tronco da árvore, continham, na base, um frasco de plástico de $1000 \mathrm{ml}$ com álcool a $92 \%$ (Fig. 1).

No dia seguinte a esses procedimentos efetuou-se, às 6:00 horas, a aplicação do inseticida durante 15 minutos. O inseticida utilizado, Lambdacialotrina a $0,5 \%$, é um piretróide sintético, não residual, vendido comercialmente como ICON, diluído em

Tabela I. Dados de altura, diâmetro do fuste na altura do peito (DAP) e diâmetro da copa das seis árvores amostradas de $A$. phalerata (Arecaceae).

\begin{tabular}{cccc}
\hline Árvore & Altura & DAP & Diâmetro da copa \\
\hline I & $7,0 \mathrm{~m}$ & $1,05 \mathrm{~m}$ & $6,0 \mathrm{~m}$ \\
II & $5,0 \mathrm{~m}$ & $2,00 \mathrm{~m}$ & $8,0 \mathrm{~m}$ \\
III & $5,0 \mathrm{~m}$ & $2,10 \mathrm{~m}$ & $3,0 \mathrm{~m}$ \\
IV & $4,0 \mathrm{~m}$ & $1,40 \mathrm{~m}$ & $5,6 \mathrm{~m}$ \\
V & $5,0 \mathrm{~m}$ & $1,80 \mathrm{~m}$ & $6,0 \mathrm{~m}$ \\
VI & $5,0 \mathrm{~m}$ & $1,40 \mathrm{~m}$ & $6,0 \mathrm{~m}$ \\
\hline
\end{tabular}


óleo diesel, sendo que para cada dois litros de óleo foram adicionados $20 \mathrm{ml}$ de inseticida, correspondendo à concentração de $0,25 \%$. Este inseticida foi lançado do chão para todas as partes da copa por meio de um pulverizador costal motorizado, Bomba Hatsuda Costal, Modelo Pulverizador UBLTipo FP4-JD22-2.T, e uma escada foi necessária para atingir as partes mais altas da copa.

Em cada árvore amostrada foi realizada uma pulverização e três coletas subseqüentes. A primeira coleta ocorreu duas horas após a aplicação do inseticida, tempo estimado para a queda dos artrópodos e recomendado para a coleta (ADIS et al.1997; Stork \& HAmmond 1997). Neste momento, as paredes dos funis receberam leves batidas para que os artrópodos caissem nos frascos coletores, sendo lavadas com auxílio de picetas com álcool a $92 \%$, e os frascos coletores trocados por outros, para a realização da segunda coleta. Em seguida, a árvore foi fortemente sacudida com auxílio de cordas amarradas em suas folhas, para que os artrópodos que ainda permaneceram presos às folhas pudessem cair nos funis. Novamente, após duas horas, foi realizada a segunda coleta, seguindo-se os mesmos procedimentos da primeira. Ao término da segunda coleta todos os funis foram retirados para a realização da última coleta.

Para a terceira e última coleta, todas as folhas do acuri foram cortadas, deixando-se apenas o ápice principal para que pudesse rebrotar. As folhas foram então lavadas com água e os artrópodos que estavam presos, coletados e acondicionados em álcool a $92 \%$.

As amostras foram transportadas para o Laboratório de Entomologia do Instituto de Biociências da Universidade Federal do Mato Grosso, triadas e identificadas ao nível de Ordem. Os adultos de Coleoptera foram separados por família, conforme LaWRenCe \& BritTon (1991), e morfoespécies, e posteriormente agrupados em guildas tróficas de acordo com ERWIN (1983b), HAMMOND et al. (1996) e ARNETT (1963), embora a prioridade para a classificação dos grupos foi a sugerida por ERWIN (1983b). Com relação aos indivíduos de Araneae, as amostras referentes às árvores I, III e IV foram enviadas a especialistas para identificação, e as obtidas das árvores II, V e VI, utilizadas para o estudo da biomassa.

O material testemunho do estudo está depositado no Laboratório de Entomologia do Instituto de Biociências da Universidade Federal do Mato Grosso, Cuiabá.

Biomassa. As árvores II, V e VI foram escolhidas aleatoriamente para o estudo de biomassa. Os táxons de artrópodos separados por árvore amostrada e por Ordem, foram secos a $60{ }^{\circ} \mathrm{C}$ até que o peso se estabilizasse, pesados em uma balança de precisão de $0,01 \mathrm{mg}$ e preservados em álcool a $92 \%$.

Análise estatística. Para testar a hipótese de diferenças na composição, abundância e freqüências das Ordens de artrópodos e morfoespécies de Coleoptera, utilizou-se a análise de variância como modelo estatístico, tendo como variável dependente a freqüência absoluta e, por fatores fixos, as árvores (I-VI), coletas (1-3) e as Ordens (1-22). Os pressupostos de aderência dos resíduos à distribuição normal (teste de

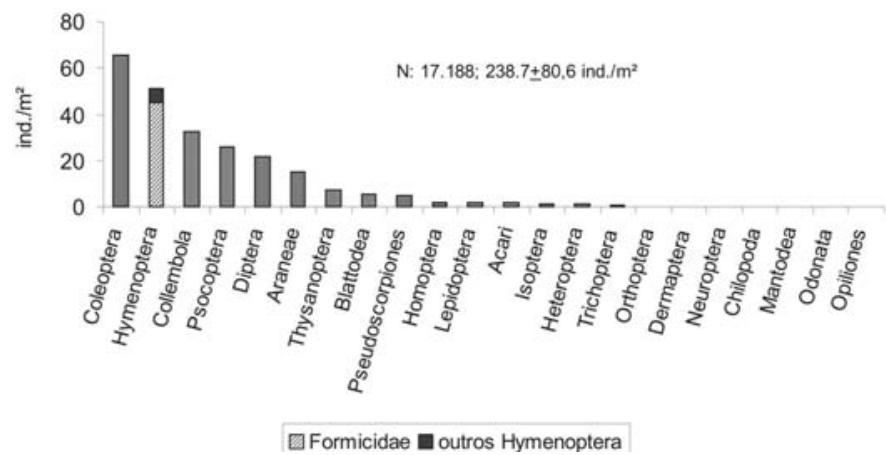

Fig. 2. Densidade (indivíduos $/ \mathrm{m}^{2}$ ) de artrópodos obtidos em copas de seis árvores amostradas de $A$. phalerata Mart. (Arecaceae). N: número total de artrópodos coletados.

Kolmogorov-Smirnov) e de homogeneidade de variâncias (teste de Levene) foram validados a um nível de significância de $0,5 \mathrm{e}$ as médias das freqüências comparadas posteriormente pelo teste de Duncan.

$\mathrm{Na}$ análise das morfoespécies de Coleoptera foram excluídas aquelas com freqüências absolutas muito baixas, ou seja, presentes em menos de três árvores, e com um número de espécimes menor que três, pois não são significativas para este tipo de análise.

O cálculo das freqüências relativas para os táxons coletados e freqüências absolutas para as famílias de Coleoptera foi feito com base no total de táxons obtidos nas seis árvores amostradas e nas três coletas efetuadas em cada uma delas.

A diversidade e riqueza de espécies da fauna de Coleoptera foi medida usando-se o índice de Shannon-Wiener (H'), e índice de riqueza de espécies Margalef (R), calculados utilizando-se Log base "e", segundo MaGURRAN (1988).

\section{RESULTADOS EDISCUSSÃO}

Em seis árvores de A. phalerata, foram coletados 17.188 artrópodos em $72 \mathrm{~m}^{2}$ de área coberta $(238,7 \pm 80,6$ indivíduos/ $\mathrm{m}^{2}$ ), distribuídos em 22 Ordens. As mais representativas foram Coleoptera $\left(27,4 \% ; 65,5 \pm 21,6\right.$ indivíduos $\left./ \mathrm{m}^{2}\right)$, Hymenoptera, a maioria Formicidae $\left(19,0 \% ; 45,3 \pm 41,6\right.$ indivíduos $\left./ \mathrm{m}^{2}\right)$, Collembola $\left(13,6 \% ; 32,4 \pm 30,3\right.$ indivíduos $\left./ \mathrm{m}^{2}\right)$, Psocoptera $\left(10,7 \% ; 25,5 \pm 7,6\right.$ indivíduos $\left./ \mathrm{m}^{2}\right)$, Diptera $(9,0 \% ; 21,6 \pm 12,1$ indivíduos $\left./ \mathrm{m}^{2}\right)$ e Araneae $\left(6,4 \% ; 15,2 \pm 5,6\right.$ indivíduos $\left./ \mathrm{m}^{2}\right)$

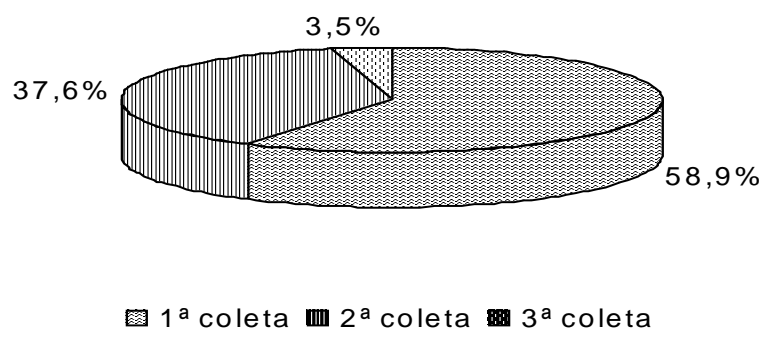

Fig. 3. Proporção de artrópodos obtidos por evento de coleta em copas de seis árvores amostradas de A. phalerata Mart. (Arecaceae). 
(Tabela II, Fig. 2).

Do total de artrópodos coletados, 10.124 indivíduos (58,9\%), correspondem à primeira coleta, $6.463(37,6 \%)$, à segunda e 601 $(3,5 \%)$ representam a terceira coleta (Fig. 3$)$.

Após a primeira coleta, aproximadamente um terço dos artrópodos ainda permaneceu na árvore, justificando a importância da realização de uma segunda amostragem. A terceira coleta, aparentemente, mostrou-se dispensável considerando a pequena proporção de indivíduos em relação às anteriores.

A freqüência da maioria dos táxons coletados foi de $100 \%$. Isoptera, Lepidoptera e Trichoptera apresentaram freqüências equivalentes a $83,3 \%$, presentes em cinco das seis árvores amostradas. Dermaptera e Neuroptera ocorreram em três árvores, com freqüência de $50 \%$, enquanto Chilopoda, Mantodea, Odonata e Opiliones com apenas 16,6\%, sendo que apenas um indivíduo de cada ordem foi coletado nas seis árvores pulverizadas.

A análise de variância ao nível de grandes táxons, na sua maioria Ordens, demonstrou não haver diferença significativa na composição da artropodofauna das seis árvores amostradas
( $\mathrm{a}=0,5 ; \mathrm{P}=0,165)$, mas sim na freqüência dos grupos coletados em cada coleta $(a=0,5 ; P=0,000)$. A comparação das médias das freqüências absolutas, por meio do teste de Duncan, mostra a predominância de seis táxons, Coleoptera, Formicidae, Collembola, Psocoptera, Diptera e Araneae (Tabela II), discutidos abaixo.

Depois dos Coleoptera, Formicidae (19,0\% do total de artrópodos) representou o segundo grupo dominante. Resultados diferentes foram obtidos nessa mesma região, quando se investigou a fauna de artrópodos em Vochysia divergens (Vochysiaceae), espécie arbórea que ocorre como monodominante no Pantanal de Mato Grosso, em que Formicidae foi predominante (MARques et al. 2001). A abundância de formigas em copas tem sido registrada por muitos estudos e parece ser característica deste ambiente em florestas tropicais, embora sejam mais abundantes que diversas (ERWIN 1983b; Adis et al.1984; WiLsON 1987; STORK 1988,1991; STORK \& Brendell 1990; Tobin 1991; Floren \& LinsenMarir 1997, 1998; HARADA \& AdIs 1997, 1998). BASSET (2001), argumentou que a causa dessa baixa riqueza de espécies pode ser explicada por enormes populações sustentadas por colônias.

Tabela II. Táxons, número de indivíduos e proporção (\%) de artrópodos coletados nas copas de seis árvores amostradas de $A$. phalerata (Arecaceae) em ordem decrescente.

\begin{tabular}{|c|c|c|c|c|c|c|c|c|}
\hline Táxon & I & II & III & IV & $\mathrm{V}$ & VI & Total & Proporção (\%) \\
\hline Coleoptera $(\mathrm{A}+\mathrm{L})$ & 430 & 765 & 1094 & 1051 & 803 & 572 & 4715 & 27,4 \\
\hline Hymenoptera & 460 & 1589 & 476 & 454 & 374 & 323 & 3676 & 21,4 \\
\hline Formicidae $(\mathrm{A}+\mathrm{L})$ & 432 & 1556 & 413 & 285 & 312 & 269 & 3267 & 19,0 \\
\hline Outros Hymenoptera & 28 & 33 & 63 & 169 & 62 & 54 & 409 & 2,4 \\
\hline Collembola & 39 & 169 & 826 & 866 & 308 & 128 & 2336 & 13,6 \\
\hline Psocoptera & 315 & 370 & 321 & 240 & 426 & 168 & 1840 & 10,7 \\
\hline $\operatorname{Diptera}(\mathrm{A}+\mathrm{L})$ & 163 & 517 & 283 & 309 & 148 & 138 & 1558 & 9,0 \\
\hline Araneae & 99 & 135 & 254 & 218 & 257 & 138 & 1101 & 6,4 \\
\hline Thysanoptera & 58 & 17 & 119 & 199 & 9 & 106 & 508 & 2,9 \\
\hline Blattodea & 8 & 21 & 149 & 104 & 52 & 57 & 391 & 2,3 \\
\hline Pseudoscorpiones & 26 & 28 & 68 & 54 & 102 & 64 & 342 & 2,0 \\
\hline Homoptera & 14 & 22 & 26 & 27 & 30 & 27 & 146 & 0,8 \\
\hline Lepidoptera $(\mathrm{A}+\mathrm{L})$ & 63 & - & 44 & 14 & 5 & 14 & 140 & 0,8 \\
\hline Acari & 21 & 27 & 40 & 20 & 6 & 16 & 130 & 0,7 \\
\hline Isoptera & 3 & 38 & 68 & 1 & - & 1 & 111 & 0,6 \\
\hline Heteroptera & 8 & 12 & 20 & 13 & 19 & 10 & 82 & 0,5 \\
\hline Trichoptera & 10 & 13 & 6 & - & 12 & 18 & 59 & 0,3 \\
\hline Orthoptera & 1 & 6 & 3 & 4 & 9 & 5 & 28 & 0,1 \\
\hline Dermaptera & 4 & - & 5 & - & - & 4 & 13 & $£ 0,1$ \\
\hline Neuroptera $(\mathrm{A}+\mathrm{L})$ & - & - & 2 & - & 3 & 3 & 8 & $£ 0,1$ \\
\hline Chilopoda & - & - & 1 & - & - & - & 1 & $£ 0,1$ \\
\hline Mantodea & - & - & - & - & 1 & - & 1 & $£ 0,1$ \\
\hline Odonata & - & - & - & - & 1 & - & 1 & $£ 0,1$ \\
\hline Opiliones & - & - & - & 1 & - & - & 1 & $£ 0,1$ \\
\hline Total & 1722 & 3729 & 3805 & 3575 & 2565 & 1792 & 17188 & 100,0 \\
\hline
\end{tabular}

$\mathrm{I}-\mathrm{VI}=$ palmeiras amostradas; $\mathrm{A}=$ adultos; $\mathrm{L}=$ larvas 
Tabela III. Táxons, número e proporção (\%) de indivíduos de Araneae obtidos em copas de três árvores de A. phalerata (Arecaceae).

\begin{tabular}{|c|c|c|}
\hline Família/Gênero/Espécie & $\mathrm{N}^{\mathrm{o}}$ de indivíduos & Proporção (\%) \\
\hline Thomisidae & 1 & 0,2 \\
\hline \multicolumn{3}{|l|}{ Theridiidae } \\
\hline Theridion (Walckenaer, 1805) & 1 & 0,2 \\
\hline Steatoda ancorata (Holmberg, 1876) & 1 & 0,2 \\
\hline Selenopidae & 23 & 4,0 \\
\hline Selenops maranhensis (Mello-Leitão, 1918) & 1 & 0,2 \\
\hline Salticidae & 220 & 38,6 \\
\hline Marma nigritarsis (Simon, 1900) & 12 & 2,1 \\
\hline Psecas (Galiano 1963) & 15 & 2,6 \\
\hline Acragas (Simon, 1900) & 1 & 0,2 \\
\hline Chira gratiosa (Soares Camargo, 1948) & 1 & 0,2 \\
\hline Formiciforme (Rainbow, 1904) & 2 & 0,3 \\
\hline Asaphobelis (Simon, 1902) & 1 & 0,2 \\
\hline Chirothecia daguerrei (Galiano, 1972) & 1 & 0,2 \\
\hline Prodidomidae & 1 & 0,2 \\
\hline Pisauridae & 6 & 1,0 \\
\hline Pholcidae & 4 & 0,7 \\
\hline Oonopidae & 10 & 1,8 \\
\hline Oonopinae & 1 & 0,2 \\
\hline Gamasomorphiinae & 28 & 5,0 \\
\hline Miturgidae & 1 & 0,2 \\
\hline Cheiracanthium inclusum (Hentz, 1847) & 2 & 0,3 \\
\hline Linyphiidae & 7 & 1,3 \\
\hline Sparassidae & 7 & 1,3 \\
\hline Polybetes rapidus (Keyserling, 1880) & 1 & 0,2 \\
\hline Gnaphosidae & 59 & 10,3 \\
\hline Vectius niger (Simon,1880) & 17 & 3,0 \\
\hline Cesonia (Hentz, 1847) & 4 & 0,7 \\
\hline Eilica aff. uniformis (Schiapelli \& Gerschmann, 1942) & 1 & 0,2 \\
\hline Eilica aff. maculatipes (Keyserling,1878) & 1 & 0,2 \\
\hline Eilica (Keyserling,1891) & 2 & 0,3 \\
\hline Ctenidae & 29 & 5,0 \\
\hline Nothroctenus aff. marschii(O.P. Cambridge 1896) & 1 & 0,2 \\
\hline Dictynidae & 7 & 1,2 \\
\hline Corinnidae & 4 & 0,7 \\
\hline Caponiidae & 3 & 0,5 \\
\hline Araneidae & 58 & 10,1 \\
\hline Metazygia gregalis (O. P. Cambridge, 1889) & 2 & 0,3 \\
\hline Anyphaenidae & 6 & 1,0 \\
\hline Jessica erythrostoma (Mello-Leitão, 1939) & 4 & 0,7 \\
\hline Amaurobiidae & 20 & 3,5 \\
\hline Goeldia (Keyserling, 1891) & 4 & 0,7 \\
\hline Total & 570 & 100,0 \\
\hline
\end{tabular}

Collembola e Psocoptera corresponderam a 24,3\% do total de artrópodos coletados, com dominância comparável à das florestas subtropicais e tropicais na Amazônia brasileira, Austrália e Indonésia (STORK \& Brendell 1993; Adis 1997; Kitching et al. 1997; Hurtado Guerrero et al. 2003). Esses dois grupos geralmente estão associados às cascas das árvores, folhas mortas, fendas e serrapilheira. Alimentam-se de matéria orgânica em decomposição, pólen, fungos, líquens, algas, restos vegetais, microepífitas ou folhas. Uma provável explicação para o grande número amostrado de Collembola e Psocoptera é o acúmulo de matéria orgânica encontrado nas bainhas de $A$. phalerata, sugerindo que os mesmos sejam, na 
verdade, habitantes destas e não da copa, confirmando a importância daqueles grupos no processo de decomposição.

Diptera correspondeu a $9,0 \%$ do total, diferindo dos resultados encontrados para $V$. divergens, onde foi o quarto grupo em número de indivíduos, com 7,4\% (MARQues et al. 2001). Em amostras de Borneo, constituiu o segundo grupo mais comum (Stork 1988). Entretanto, em Sulawesi, correspondeu a cerca de $50 \%$ da fauna obtida com aplicação de inseticida, chegando a representar mais que $80 \%$ em algumas coletas (STORK \& BRENDELL 1990).

Embora Diptera seja freqüentemente um dos grupos mais abundantes em amostragens de artrópodos em copas, é pouco considerado em estudos taxonômicos, apesar de constituir fonte de significantes interações de espécies neste habitat (Didham 1997). Dados obtidos em copas de duas árvores de Goupia glabra Aublet. (Celastraceae), na Amazônia, sugerem uma interação biótica entre Formicidae e Cecydomyiidae que constróem galhas (Adis et al.1998a).

Os indivíduos de Araneae corresponderam a 6,4\% da fauna total, distribuídos em 18 famílias, 3 subfamílias, 7 gêneros e 13 espécies, sendo Salticidae, Gnaphosidae, Araneidae, Oonopidae e Ctenidae as mais representativas (Tabelas II, III, Fig. 4). O número proporcionalmente baixo de espécies reflete a grande quantidade de indivíduos jovens (cerca de 30\% da amostra) e a conseqüente impossibilidade de identificação ao nível de espécie. Outro problema encontrado quanto à identificação, é a existência de grupos não estudados na Região Neotropical, ou mesmo de gêneros de algumas famílias como Oonopidae, Pholcidae e Salticidae, o que dificulta a identificação das espécies (BRESCOVIT, comunicação pessoal; BASSET 2001). Estudos realizados em copas de duas árvores de Goupia glabra Aublet. (Celastraceae) na Amazônia Central mostraram um total de 142 indivíduos de Araneae, distribuidos em 21 famílias, sendo Araneidae (12 spp.), Theridiidae (12 spp.) e Salticidae (10 spp.), as mais abundantes (HöFER et al. 1994).

\section{Biomassa}

A biomassa total de artrópodos amostrada para três árvores de $A$. phalerata (II,V,VI), correspondeu a $15,1 \mathrm{~g}$ de peso seco e $0,4 \mathrm{mg} / \mathrm{m}^{2}(0,13 \pm 0,04 /$ árvore $)$. A tabela IX representa a biomassa

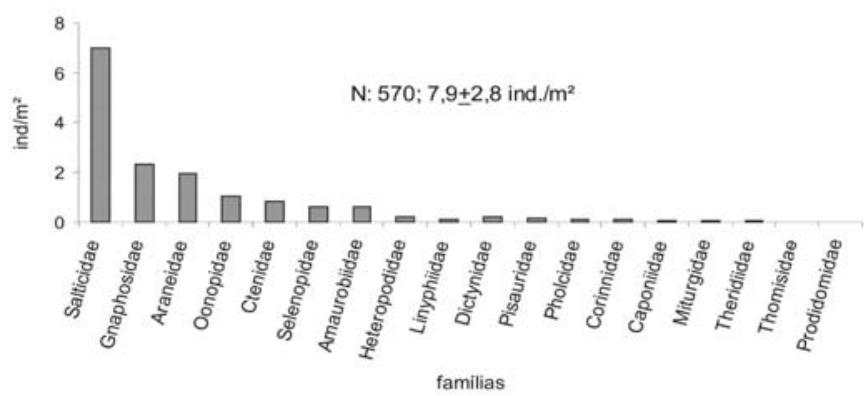

Fig. 4. Densidade (indivíduos $/ \mathrm{m}^{2}$ ) das famílias de Araneae, coletadas em copas de três árvores amostradas de A. phalerata Mart. (Arecaceae). N: número total de aranhas coletadas. dos táxons em ordem descendente de peso seco. As quatro ordens com maior peso seco foram Coleoptera $\left(0,25 \mathrm{mg} / \mathrm{m}^{2}\right.$; $0,08 \pm 0,03$ /árvore), Blattodea $\left(0,05 \mathrm{mg} / \mathrm{m}^{2} ; 0,017 \pm 0,0009\right.$ /árvore $)$, Formicidae $\left(0,04 \mathrm{mg} / \mathrm{m}^{2} ; 0,012 \pm 0,0043\right.$ /árvore $)$ e Orthoptera $(0,03$ $\mathrm{mg} / \mathrm{m}^{2}, 0,010 \pm 0,007 /$ árvore) que, juntas, corresponderam a $90,3 \%$ da biomassa total, sendo que $61,3 \%$ refere-se a Coleoptera (Tabela IX).

Dentre essas ordens, Coleoptera teve maior peso por apresentar élitros esclerotizados e, nos Curculionidae, rostros córneos, além de indivíduos com corpos maiores como, por exemplo, nas famílias Tenebrionidae, Scarabaeidae e Carabidae. Apesar de poucos indivíduos terem sido coletados, a ordem Blattodea obteve uma biomassa representativa, pois apresentou indivíduos com corpos e tégminas bem desenvolvidos. O mesmo ocorreu com Orthoptera.

O grande número de indivíduos da família Formicidae (1.556) coletados na palmeira II (Tabela II) associado ao grande tamanho é, provavelmente, a explicação para uma biomassa tão representativa em Hymenoptera.

Os dados de biomassa evidenciam uma relação entre tamanho e peso dos indivíduos, necessitando, portanto, de estudos a respeito do tamanho corporal aliado aos hábitos alimentares dos grupos. Segundo BASSET et al. (1994), estas discussões proporcionam a compreensão mais ampla sobre a relação entre biomassa e estrutura de comunidades de artrópodos na copa.

\section{Coleoptera}

Famílias e morfoespécies. Foram obtidos 4.715 indivíduos adultos de Coleoptera, representando 326 morfoespécies distribuídas em 48 famílias. As mais abundantes foram Tenebrionidae $(22,9 \%)$, Curculionidae $(22,0 \%)$, Carabidae $(10,9 \%)$ e Staphylinidae (7,9\%), compondo $63,7 \%$ do total coletado. Curculionidae (44 spp.), Staphylinidae (40 spp.) e Chrysomelidae (34 spp.), apresentaram uma riqueza maior de morfoespécies, enquanto para Tenebrionidae e Carabidae foram identificadas 17 e 13 morfoespécies, respectivamente (Tabela IV, Fig. 5). Do total de espécimes de Coleoptera, apenas 47 $(1,0 \%)$ não foram identificados (Tabela IV) devido à perda de estruturas morfológicas fundamentais para identificação.

Indivíduos da família Curculionidae foram identificados em 12 subfamílias, 11 tribos, 9 gêneros e 8 espécies, sendo Celetes sp. o gênero com maior número de indivíduos coletados (562) (Tabela VII).

Singletons. Espécies denominadas "singletons" são definidas como raras e representam mais da metade do total obtido em estudos de comunidades de insetos em florestas tropicais úmidas (Allison et al. 1997; NovotNy \& BASSET 2000).

Dentre as 326 morfoespécies identificadas, 255 (78,2\%), apresentaram menos que dez indivíduos, $34(10,4 \%)$ foram "doubletons", e 136 (41,7\%) espécies "singletons" (Fig. 5). A porcentagem de "singletons" por árvore variou entre 36,7\% e 48,4\% (Tabela V). As famílias Chrysomelidae (25 spp.) e 
Tabela IV. Número de morfoespécies, indivíduos e proporção (\%) das famílias de Coleoptera (adultos) obtidos nas copas de seis árvores de $A$. phalerata (Arecaceae), com indicação de guildas tróficas. H (herbívoros), D (decompositores), $\mathrm{P}$ (predadores), $\mathrm{F}$ (fungívoros) e $\mathrm{S}$ (saprófagos).

\begin{tabular}{|c|c|c|c|c|}
\hline Família & $\mathrm{N}^{\mathrm{o}}$ de indivíduos & $\mathrm{n}^{\mathrm{o}}$ de morfoespécies & Proporção \% & Guildas \\
\hline Tenebrionidae & 1080 & 17 & 22,9 & $\mathrm{~S}(\mathrm{~F})$ \\
\hline Curculionidae & 1039 & 44 & 22,0 & $\mathrm{H}$ \\
\hline Carabidae & 511 & 13 & 10,9 & $\mathrm{P}$ \\
\hline Staphylinidae & 374 & 40 & 7,9 & $\mathrm{P}(\mathrm{S}, \mathrm{F})$ \\
\hline Phalacridae & 271 & 7 & 5,7 & $\mathrm{H}$ \\
\hline Scarabaeidae & 216 & 5 & 4,6 & $\mathrm{H}(\mathrm{S})$ \\
\hline Anobiidae & 167 & 6 & 3,5 & $\mathrm{H}(\mathrm{F})$ \\
\hline Nitidulidae & 165 & 10 & 3,5 & $\mathrm{~S}$ \\
\hline Lathridiidae & 158 & 5 & 3,3 & $\mathrm{~F}$ \\
\hline Chrysomelidae & 76 & 34 & 1,6 & $\mathrm{H}$ \\
\hline Alleculidae & 69 & 3 & 1,5 & $\mathrm{H}$ \\
\hline Pselaphidae & 56 & 4 & 1,2 & $\mathrm{~F}$ \\
\hline Biphylidae & 45 & 4 & 1,0 & $\mathrm{~F}$ \\
\hline Elateridae & 45 & 14 & 1,0 & $\mathrm{H}(\mathrm{P})$ \\
\hline Colydiidae & 42 & 8 & 0,9 & $\mathrm{P}(\mathrm{F})$ \\
\hline Cucujidae & 35 & 9 & 0,7 & $\mathrm{P}(\mathrm{F})$ \\
\hline Trogositidae & 33 & 2 & 0,7 & $\mathrm{P}(\mathrm{S})$ \\
\hline Hydraenidae & 28 & 5 & 0,6 & $\mathrm{P}$ \\
\hline Corylophidae & 25 & 4 & 0,5 & $\mathrm{P}$ \\
\hline Histeridae & 23 & 4 & 0,5 & $\mathrm{P}$ \\
\hline Endomychidae & 22 & 3 & 0,5 & $\mathrm{~F}$ \\
\hline Cerambycidae & 22 & 9 & 0,5 & $\mathrm{H}$ \\
\hline Bruchidae & 18 & 11 & 0,4 & $\mathrm{H}$ \\
\hline Dytiscidae & 18 & 8 & 0,4 & $\mathrm{P}$ \\
\hline Hydrophilidae & 16 & 6 & 0,4 & $\mathrm{~S}$ \\
\hline Bothrideridae & 14 & 2 & 0,3 & $\mathrm{P}$ \\
\hline Scolytidae & 13 & 7 & 0,3 & $\mathrm{H}(\mathrm{F})$ \\
\hline Meloidae & 13 & 1 & 0,3 & $\mathrm{H}$ \\
\hline Anthicidae & 10 & 5 & 0,2 & S \\
\hline Monommidae & 9 & 1 & 0,2 & $\mathrm{H}$ \\
\hline Lampyridae & 9 & 4 & 0,2 & $\mathrm{P}$ \\
\hline Scydmaenidae & 8 & 4 & 0,1 & $\mathrm{P}$ \\
\hline Cleridae & 6 & 4 & 0,1 & $\mathrm{P}$ \\
\hline Platypodidae & 5 & 3 & 0,1 & $\mathrm{~F}$ \\
\hline Languriidae & 4 & 1 & 0,09 & $\mathrm{H}$ \\
\hline Ptiliidae & 4 & 2 & 0,09 & $\mathrm{~S}(\mathrm{~F})$ \\
\hline Mordelidae & 3 & 3 & 0,06 & $\mathrm{H}$ \\
\hline Erotylidae & 3 & 1 & 0,06 & $\mathrm{~F}$ \\
\hline Apionidae & 2 & 2 & 0,05 & $\mathrm{H}$ \\
\hline Buprestidae & 2 & 2 & 0,05 & $\mathrm{H}$ \\
\hline Coccinellidae & 2 & 2 & 0,05 & $\mathrm{P}$ \\
\hline Silvanidae & 1 & 1 & 0,02 & $\mathrm{~F}$ \\
\hline Rhizophagidae & 1 & 1 & 0,02 & $\mathrm{P}$ \\
\hline Noteridae & 1 & 1 & 0,02 & $\mathrm{P}$ \\
\hline Cantharidae & 1 & 1 & 0,02 & $\mathrm{H}$ \\
\hline Leiodidae & 1 & 1 & 0,02 & $\mathrm{~S}$ \\
\hline Phengodidae & 1 & 1 & 0,02 & $\mathrm{P}$ \\
\hline Mycetophagidae & 1 & 1 & 0,02 & $\mathrm{H}$ \\
\hline Não identificado & 47 & - & 1,0 & - \\
\hline Total & 4715 & 326 & 100,0 & \\
\hline
\end{tabular}


Tabela V. Número total de morfoespécies, indivíduos, "singletons" e sua proporção (\%), da ordem Coleoptera (adultos) obtidos nas copas de seis árvores de $A$. phalerata (Arecaceae).

\begin{tabular}{lcccccc}
\hline Palmeira & I & II & III & I & V & V \\
$\mathrm{N}^{\circ}$ de morfoespécies (S) & 85 & 94 & 163 & 127 & 103 & 90 \\
$\mathrm{~N}^{\circ}$ de indivíduos (N) & 790 & 566 & 424 & 759 & 1083 & 1046 \\
Razão (N/S) & 9,3 & 6,0 & 5,6 & 6,0 & 10,5 & 11,6 \\
Proporção de "Singletons" (\%) & 38,8 & 38,3 & 48,4 & 40,9 & 44,6 & 36,7 \\
$\mathrm{~N}^{\circ}$ de morfoespécies abundantes & 4 & 8 & 9 & 8 & 10 & 7 \\
\hline
\end{tabular}

Staphylinidae (16 spp.) contribuíram com maior número de morfoespécies "singletons".

Uma explicação para o elevado número de "singletons", refere-se a problemas com replicações de amostragens sazonais ou espaciais que são muitas vezes insuficientes, resultando em numerosas espécies aparentemente raras. Novotny \& BASSET (2000) argumentaram que este problema é resultado da inadequação na padronização de métodos de coletas massivas com inseticida, armadilhas de luz ou Malaise, além da dificuldade em saber com precisão em qual planta hospedeira o indivíduo foi coletado. Essa situação é verificada neste estudo, pois a coleta foi pontual empregando-se um método de coleta massiva.

A riqueza de comunidades de herbívoros sobre plantas e, particularmente, o número de espécies raras pode ser incrementado por um constante influxo de espécies oriundas de plantas adjacentes, explicado pela teoria de "efeito massa", proposta por SHMIDA \& WILSON (1985). Esse influxo é importante para insetos em florestas úmidas, onde numerosas espécies de plantas crescem muito próximas. Com relação a essa teoria, é possível que o influxo de espécies não incremente consideravelmente a riqueza de espécies em $A$. phalerata, considerando que esta palmeira ocupa extensas áreas no Pantanal de Mato Grosso, ocorrendo como monodominante em uma associação conhecida como acurizal.

Guildas tróficas. Segundo as classificações propostas por ERwin (1983b), Hammond et al. (1996) e Arnett (1963), cinco

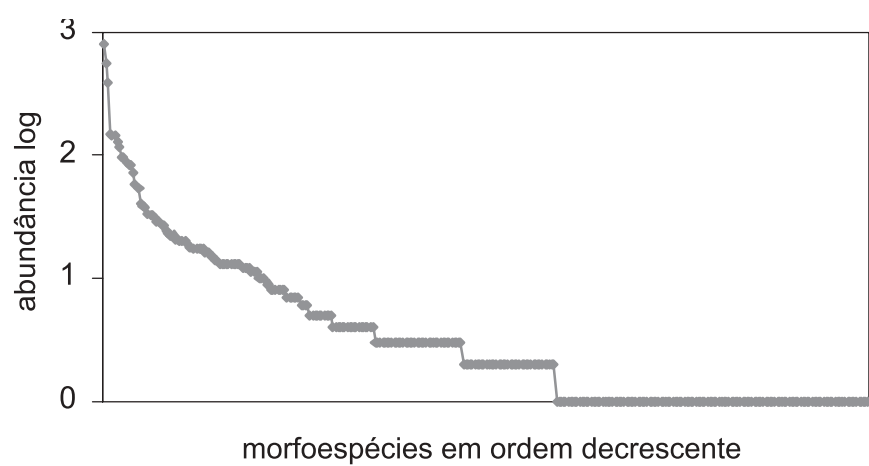

Fig. 5. Abundância relativa em ordem decrescente das morfoespécies de Coleoptera, em escala logarítmica. guildas tróficas foram reconhecidas para os Coleoptera, com predominância de herbívoros, seguida de predadores, fungívoros e saprófagos (Fig. 6).

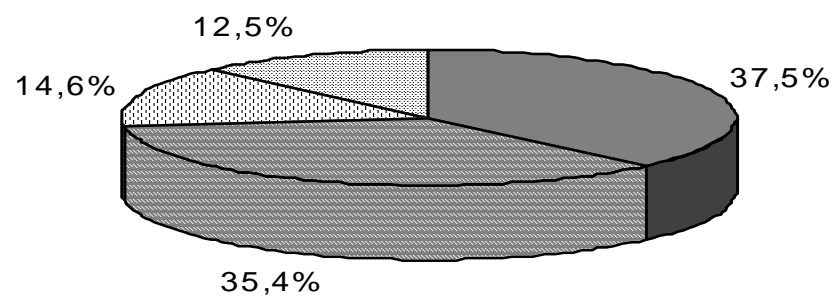

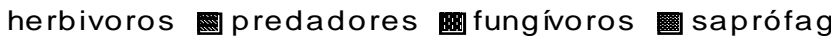

Fig. 6. Porcentagem das guildas tróficas em Coleoptera (adultos), obtidas em copas de seis árvores amostradas de A phalerata Mart. (Arecaceae).

Em A. phalerata, os herbívoros dominaram devido ao grande número de indivíduos das famílias Curculionidae, Phalacridae, Scarabaeidae e Anobiidae que, somados, correspondem a $35,8 \%$ de besouros coletados; os predadores foram representados por Carabidae (10,9\%) e Staphylinidae $(7,9 \%)$, os fungívoros, por Lathridiidae $(3,3 \%)$, Pselaphidae $(1,2 \%)$ e Biphylidae ( $1,0 \%)$ e os decompositores, na sua maioria, por Tenebrionidae $(22,9 \%)$ (Tabela IV).

Estes dados são semelhantes aos obtidos na Venezuela, em que houve predominância de herbívoros seguidos de fungívoros e predadores (DAvies et al. 1997) e aos de Ruanda, com abundância de herbívoros e fungívoros (WAGNER 1997). O mesmo foi observado no Pantanal de Mato Grosso (MARQues et al. (2001), em que os herbívoros dominaram sobre xilófagos, saprófagos, predadores e fungívoros, diferindo daqueles da Austrália e Sulawesi (HAMmOND 1990; HAMmOND et al. 1996) em que predadores foram dominantes.

ERwin (1983b) observou que, em florestas tropicais na Amazônia, os Tenebrionidae são os decompositores predominantes, os Anthribidae e Endomychidae constituem os fungívoros dominantes e Coccinellidae e Staphylinidae, os predadores dominantes. Em termos de tamanho, Scarabaeidae são os maiores herbívoros, Tenebrionidae os maiores decompositores, Anthribidae os maiores fungívoros e Lampyridae, os grandes predadores. 
Tabela VI. Número de indivíduos e freqüência absoluta de Coleoptera (adultos) por famílias obtidas nas copas de seis árvores de A. phalerata Mart. (Arecaceae).

\begin{tabular}{|c|c|c|c|c|c|c|c|c|}
\hline Família & I & II & III & IV & $\mathrm{V}$ & VI & Total & Frequência $(\%)$ \\
\hline Alleculidae & 5 & 14 & 1 & 3 & 37 & 9 & 69 & 100,0 \\
\hline Anobiidae & 9 & 10 & 25 & 83 & 31 & 9 & 167 & 100,0 \\
\hline Anthicidae & 0 & 2 & 7 & 0 & 0 & 1 & 10 & 50,0 \\
\hline Apionidae & 0 & 0 & 0 & 2 & 0 & 0 & 2 & 16,6 \\
\hline Biphylidae & 4 & 3 & 13 & 3 & 11 & 11 & 45 & 100,0 \\
\hline Bothrideridae & 1 & 5 & 0 & 8 & 0 & 0 & 14 & 50,0 \\
\hline Bruchidae & 1 & 8 & 2 & 2 & 5 & 0 & 18 & 83,3 \\
\hline Buprestidae & 1 & 0 & 1 & 0 & 0 & 0 & 2 & 33,3 \\
\hline Cantharidae & 0 & 0 & 0 & 0 & 0 & 1 & 1 & 16,6 \\
\hline Carabidae & 49 & 17 & 193 & 58 & 131 & 63 & 511 & 100,0 \\
\hline Cerambycidae & 3 & 1 & 3 & 3 & 1 & 11 & 22 & 100,0 \\
\hline Chrysomelidae & 6 & 14 & 10 & 17 & 14 & 15 & 76 & 100,0 \\
\hline Cleridae & 1 & 2 & 3 & 0 & 0 & 0 & 6 & 50,0 \\
\hline Colydiidae & 5 & 1 & 4 & 8 & 21 & 3 & 42 & 100,0 \\
\hline Coccinellidae & 0 & 0 & 0 & 1 & 0 & 1 & 2 & 33,3 \\
\hline Corylophidae & 1 & 1 & 11 & 0 & 11 & 1 & 25 & 83,3 \\
\hline Cucujidae & 4 & 0 & 0 & 1 & 11 & 19 & 35 & 66,6 \\
\hline Curculionidae & 51 & 137 & 126 & 497 & 94 & 134 & 1039 & 100,0 \\
\hline Dytiscidae & 4 & 0 & 13 & 1 & 0 & 0 & 18 & 50,0 \\
\hline Elateridae & 7 & 5 & 7 & 7 & 9 & 10 & 45 & 100,0 \\
\hline Endomychidae & 7 & 3 & 5 & 1 & 1 & 5 & 22 & 100,0 \\
\hline Erotylidae & 1 & 1 & 0 & 0 & 1 & 0 & 3 & 50,0 \\
\hline Histeridae & 6 & 0 & 1 & 1 & 6 & 9 & 23 & 83,3 \\
\hline Hydraenidae & 4 & 2 & 19 & 3 & 0 & 0 & 28 & 66,6 \\
\hline Hydrophilidae & 0 & 1 & 4 & 9 & 1 & 1 & 16 & 83,3 \\
\hline Lampyridae & 1 & 3 & 1 & 4 & 0 & 0 & 9 & 66,6 \\
\hline Languriidae & 2 & 0 & 2 & 0 & 0 & 0 & 4 & 33,3 \\
\hline Lathridiidae & 39 & 52 & 44 & 12 & 8 & 3 & 158 & 100,0 \\
\hline Leiodidae & 0 & 0 & 0 & 0 & 0 & 1 & 1 & 16,6 \\
\hline Meloidae & 8 & 2 & 1 & 0 & 1 & 1 & 13 & 83,3 \\
\hline Monnomidae & 0 & 7 & 0 & 0 & 2 & 0 & 9 & 33,3 \\
\hline Mordelidae & 1 & 0 & 0 & 1 & 0 & 1 & 3 & 50,0 \\
\hline Mycetophagidae & 0 & 0 & 1 & 0 & 0 & 0 & 1 & 16,6 \\
\hline Nitidulidae & 4 & 31 & 28 & 12 & 3 & 87 & 165 & 100,0 \\
\hline Noteridae & 0 & 0 & 1 & 0 & 0 & 0 & 1 & 16,6 \\
\hline Phalacridae & 42 & 62 & 89 & 9 & 55 & 14 & 271 & 100,0 \\
\hline Phengodidae & 1 & 0 & 0 & 0 & 0 & 0 & 1 & 16,6 \\
\hline Platypodidae & 0 & 2 & 2 & 0 & 0 & 1 & 5 & 50,0 \\
\hline Pselaphidae & 2 & 10 & 18 & 20 & 1 & 5 & 56 & 100,0 \\
\hline Ptiliidae & 2 & 1 & 0 & 1 & 0 & 0 & 4 & 50,0 \\
\hline Rhizophagidae & 0 & 0 & 1 & 0 & 0 & 0 & 1 & 16,6 \\
\hline Scarabaeidae & 1 & 3 & 44 & 39 & 99 & 30 & 216 & 100,0 \\
\hline Scolytidae & 0 & 0 & 0 & 0 & 10 & 3 & 13 & 33,3 \\
\hline Scydmaenidae & 0 & 0 & 6 & 2 & 0 & 0 & 8 & 33,3 \\
\hline Silvanidae & 0 & 0 & 1 & 0 & 0 & 0 & 1 & 16,6 \\
\hline Staphylinidae & 27 & 87 & 108 & 80 & 22 & 50 & 374 & 100,0 \\
\hline Tenebrionidae & 123 & 264 & 291 & 152 & 204 & 46 & 1080 & 100,0 \\
\hline Trogositidae & 1 & 7 & 0 & 5 & 1 & 19 & 33 & 83,3 \\
\hline Não identificado & 6 & 7 & 8 & 6 & 12 & 8 & 47 & - \\
\hline Total & 430 & 765 & 1094 & 1051 & 803 & 572 & 4715 & - \\
\hline
\end{tabular}

$\mathrm{N}^{\text {os }} \mathrm{I}$-VI correspondem às palmeiras amostradas. 
Tabela VII. Táxons, número de indivíduos e proporção (\%) de Curculionidae (Coleoptera) em copas de seis árvores de $A$. phalerata Mart. (Arecaceae).

\begin{tabular}{|c|c|c|}
\hline Subfamília/Tribo/Gênero/Espécie & Total & Proporção (\%) \\
\hline \multicolumn{3}{|l|}{ Baridinae, Coleonertina } \\
\hline Coleonertus cf. silvestris Casey, 1922 & 18 & 1,7 \\
\hline Baridinae sp.08 & 3 & 0,3 \\
\hline Baridinae sp.09 & 1 & 0,1 \\
\hline \multicolumn{3}{|l|}{ Baridinae, Centrinini } \\
\hline Centrinites $\mathbf{s p} .01$ & 19 & 1,8 \\
\hline \multicolumn{3}{|l|}{ Baridinae, Madarini } \\
\hline Notesiaspis hystrix Casey, 1922 & 13 & 1,2 \\
\hline Madarini sp.01 & 11 & 1,0 \\
\hline Madarini sp.05 & 3 & 0,3 \\
\hline Madarini sp.06 & 13 & 1,2 \\
\hline Madarini sp.08 & 2 & 0,2 \\
\hline \multicolumn{3}{|l|}{ Ceotorrhynchinae } \\
\hline Ceotorrhynchinae sp.01 & 33 & 3,2 \\
\hline Ceotorrhynchinae sp.14 & 6 & 0,6 \\
\hline \multicolumn{3}{|l|}{ Cossoninae } \\
\hline Stenomimus sp.01 & 3 & 0,3 \\
\hline Stenomimus sp.02 & 13 & 1,2 \\
\hline Stenopelmini $\mathbf{s p} .32$ & 3 & 0,3 \\
\hline \multicolumn{3}{|l|}{ Cryptorhynchinae, Cryptorhynchini } \\
\hline Phyrdenus sp.01 & 3 & 0,3 \\
\hline Cryptorhynchinae sp.14 & 7 & 0,7 \\
\hline Cryptorhynchinae sp. 15 & 40 & 3,8 \\
\hline Cryptorhynchinae sp.16 & 1 & 0,1 \\
\hline Cryptorhynchinae sp. 17 & 17 & 1,6 \\
\hline \multicolumn{3}{|l|}{ Curculionidae } \\
\hline Curculionidae sp.04 & 5 & 0,5 \\
\hline \multicolumn{3}{|l|}{ Erirhininae, Derelomini } \\
\hline Celetes sp. 01 & 9 & 0,9 \\
\hline Celetes sp. 02 & 553 & 53,2 \\
\hline Andrathobius sp.02 & 87 & 8,4 \\
\hline Derelomini sp. 14 & 12 & 1,2 \\
\hline Derelomini sp. 15 & 3 & 0,3 \\
\hline Derelomini sp.16 & 3 & 00,3 \\
\hline \multicolumn{3}{|l|}{ Erirhininae, Stenopelmini } \\
\hline Notiodes inaequalis (Hustache, 1826) & 21 & 2,0 \\
\hline Notiodes nanus Boheman, 1859 & 3 & 0,3 \\
\hline \multicolumn{3}{|l|}{ Molytinae } \\
\hline Molytinae sp.01 & 3 & 0,3 \\
\hline Molytinae sp.02 & 5 & 0,5 \\
\hline \multicolumn{3}{|l|}{ Molytinae, Cholini } \\
\hline Homalinotus depressus (L., 1764) & 8 & 0,8 \\
\hline Homalinotus validus (Olivier, 1790) & 3 & 0,3 \\
\hline \multicolumn{3}{|l|}{ Molytinae, Conotrachelini } \\
\hline Conotrachelus sp.09 & 3 & 0,3 \\
\hline \multicolumn{3}{|l|}{ Otidocephalinae, Otidocephalini } \\
\hline Myrmex sp.01 & 3 & 0,3 \\
\hline \multicolumn{3}{|l|}{ Petalochilinae } \\
\hline Petalochilus sp. 01 & 11 & 1,0 \\
\hline \multicolumn{3}{|l|}{ Polydrosinae, Naupactini } \\
\hline Parapantomorus fluctuosus (Boheman, 1840) & 15 & 1,5 \\
\hline Naupactus tarsalis Boheman, 1840 & 22 & 2,1 \\
\hline Naupactini sp.03 & 40 & 3,8 \\
\hline Naupactini sp.04 & 3 & 0,3 \\
\hline \multicolumn{3}{|l|}{ Rhynchophorinae } \\
\hline Rhynchophorinae sp.01 & 3 & 0,3 \\
\hline Tychiinae, Tychiini & & \\
\hline Sibinia sp.01 & 4 & 0,4 \\
\hline Sibinia sp.02 & 3 & 0,3 \\
\hline Zygopinae, Lechriopini & & \\
\hline Lechriopini sp. 05 & 5 & 0,5 \\
\hline Lechriopini sp.06 & 3 & 0,3 \\
\hline Total & 1039 & 100,0 \\
\hline
\end{tabular}


Tabela VIII. Índices de diversidade calculados para a comunidade de Coleoptera obtida nas copas de seis árvores A. phalerata Mart. (Arecaceae).

\begin{tabular}{cccc}
\hline $\begin{array}{c}\text { Índice de Shannon- } \\
\text { Wiener }(\mathrm{H})\end{array}$ & "Evenness" & $\begin{array}{c}\text { Índice de Riqueza de espécies } \\
\text { de Margalef }\end{array}$ & $\mathrm{N}^{\text {o de morfoespécies }}$ \\
\hline 4,55 & 0,79 & 38,7 & 326 \\
\hline
\end{tabular}

Análise de variância. A tabela VI mostra que 34 famílias tiveram freqüências elevadas, estando presentes em pelo menos três das seis árvores, e apenas 14 com freqüências menores, presentes em menos que três. Estes dados diferem daqueles de Allison et al. (1997), que também analisaram apenas uma espécie de árvore em Papua Nova Guiné e encontraram metade dos indivíduos de Coleoptera (55,3\%) sobre apenas uma árvore e menos que um terço sobre três ou mais árvores.

A análise de variância demonstrou não haver diferença significativa na composição da fauna de Coleoptera nas seis árvores amostradas $(\mathrm{a}=0,5 ; \mathrm{P}=0,123)$, mas sim na distribuição das freqüências absolutas das morfoespécies $(\mathrm{a}=0,5 ; \mathrm{P}=0,000)$. Apesar da não aderência à distribuição normal, o teste de Levene demonstra que as variâncias das amostras são homogêneas $(\mathrm{a}=0,5 ; \mathrm{P}=0,958)$. Isto se deve à presença de três morfoespécies das famílias Tenebrionidae, Curculionidae e Carabidae que apresentaram mais de 300 indivíduos.
Riqueza e diversidade de espécies. O índice de diversidade de espécies de Shannon-Wiener (H') calculado para a comunidade de Coleoptera é influenciado pelo grande número de espécies "singletons" (136; 41,7\%), sendo que o "evenness" demonstra uma uniformidade alta na distribuição das espécies dentro desta comunidade (Tabela VIII).

\section{CONCLUSÕES}

A análise de variância ao nível de grandes táxons como famílias de Coleoptera, demonstrou não haver diferença significativa na composição da artropodofauna nas seis árvores amostradas de $A$. phalerata, mas sim na freqüência das Ordens, embora a maioria destas tenha estado presente em todas as árvores investigadas.

Isto indica que o número de árvores amostradas e eventos de coleta efetuados podem ser considerados significativos para

Tabela IX. Biomassa total e proporção (\%) dos táxons de artrópodos obtidos em três copas de A. phalerata Mart. (Arecaceae).

\begin{tabular}{|c|c|c|}
\hline Táxons & Peso (mg) & Proporção (\%) \\
\hline Coleoptera & 9,2771 & 61,36 \\
\hline Blattodea & 1,8698 & 12,37 \\
\hline Hymenoptera & 1,4513 & 9,61 \\
\hline Formicidae & 1,3876 & 9,18 \\
\hline Outros Hymenoptera & 0,0637 & 0,43 \\
\hline Orthoptera & 1,1299 & 7,48 \\
\hline Araneae & 0,8212 & 5,43 \\
\hline Diptera & 0,1322 & 0,88 \\
\hline Homoptera & 0,1057 & 0,70 \\
\hline Lepidoptera & 0,0687 & 0,46 \\
\hline Odonata & 0,0590 & 0,40 \\
\hline Heteroptera & 0,0546 & 0,36 \\
\hline Psocoptera & 0,0391 & 0,26 \\
\hline Pseudoscorpiones & 0,0334 & 0,22 \\
\hline Isoptera & 0,0329 & 0,22 \\
\hline Acari & 0,0161 & 0,11 \\
\hline Trichoptera & 0,0125 & 0,08 \\
\hline Collembola & 0,0075 & 0,05 \\
\hline Mantodea & 0,0040 & 0,02 \\
\hline Thysanoptera & 0,0029 & 0,01 \\
\hline Neuroptera & 0,0017 & 0,01 \\
\hline Dermaptera & 0,0010 & $<0,05$ \\
\hline Total & $15,1206 \mathrm{~g}$ & 100,00 \\
\hline
\end{tabular}


concluir que os táxons obtidos representam a comunidade de artrópodos em copa de $A$. phalerata, durante o período de seca do Pantanal de Mato Grosso.

As ordens predominantes para este estudo como Coleoptera, Formicidae, Collembola, Psocoptera, Diptera e Araneae corroboram os dados de pesquisas realizadas em copas de florestas tropicais e subtropicais (BASSET 2001), indicando um padrão de dominância destes grupos nestas regiões.

O mesmo padrão de dominância pode ser verificado com relação à riqueza de espécies entre as famílias de Coleoptera. Curculionidae, Staphylinidae e Chrysomelidae são as mais ricas em espécies em copas de florestas tropicais e subtropicais (BASSET 2001), dominância também encontrada em $A$. phalerata.

A biomassa foi melhor avaliada em grupos de artrópodos com corpos mais pesados como Coleoptera, Hymenoptera, Blattodea, Formicidae e Orthoptera e nas famílias de Coleoptera, Curculionidae, Tenebrionidae, Scarabaeidae e Carabidae.

A alta abundância de artrópodos encontrada em $A$. phalerata demonstra que esta espécie vegetal é utilizada por grupos de artrópodos como fonte de recurso alimentar, local de reprodução e abrigo.

O acúmulo de matéria orgânica nas bainhas foliares de $A$. phalerata propicia o surgimento de um microhabitat importante principalmente para grupos como Psocoptera e Collembola, essencialmente decompositores.

Agradecimentos. Este estudo é resultado da cooperação científica entre o Instituto Max-Planck para Limnologia, Plön, Alemanha e Universidade Federal de Mato Grosso - Cuiabá, Programa SHIFT (Studies of Human Impact on Forests and Floodplains in the Tropics), financiado pelo Bundesministerium für Bildung, Wissenschaft Forschung und Technologie (BMBF), Conselho Nacional de Desenvolvimento Científico e Tecnológico (CNPq) e Instituto Brasileiro de Meio Ambiente e Recursos Naturais Renováveis (IBAMA). Agradecemos à Fundação Nacional de Saúde (FNS-Cuiabá-Mato Grosso) e ao Sr. João Bosco, gerente da Fazenda Experimental (Faculdade de Agronomia e Medicina Veterinária Universidade Federal de Mato Grosso), pelo empréstimo do pulverizador costal para as atividades de coleta. Aos técnicos Francisco Gonçalves de Assis Rondon (Universidade Federal de Mato Grosso), Benedito Antonio Martins (Fundação Nacional de Saúde) e alunos do Laboratório de Entomologia do Instituto de Biociências da Universidade Federal de Mato Grosso pelo auxílio nas coletas de campo. Ao Coronel Nelson Prado Godoy, Diretor do Parque da Aeronáutica - Lagoa Santa -Minas Gerais e ao Sargento Luis Carlos, pela doação dos pára-quedas para confecção dos funis coletores. Dr. Sérgio Vanin (Museu de Zoologia da Universidade de São Paulo) e Dr. Antônio Brescovit (Instituto Butantã -São Paulo) pela identificação dos Curculionidae e Araneae, respectivamente.

\section{REFERÊNCIAS}

AdÁmoli, J. A. 1986. Fitogeografia do Pantanal, p. 105-106. Anais do I Simpósio sobre Recursos Naturais e Sócio-Econômicos do Pantanal. Brasília, EMBRAPA-DDT.

ADIs, J. 1997. Terrestrial invertebrates: survival strategies, group spectrum, dominance and activity patterns, vol. 126, p. 318-330. In: W. J. JUnK (ed.) The central Amazon floodplain. Ecological Studies. Berlin, 525 p.

Adis, J.; Y. D. Lubin \& G. G. Montgomery. 1984. Arthropods from the canopy inundated and terra firme forests near Manaus, Brazil, with critical considerations on the pyrethrum-fogging technique. Studies on Neotropical Fauna and Environment 19(4): 223-236.
Adis, J.; W. Paarmann; C. R. V. da Fonseca \& J. A. Rafael. 1997. Knockdown efficiency of natural pyrethrum and survival rate of living arthropods obtained by canopy fogging in Central Amazonia, p. 67-81. In: N. E. Stork; J. Adis \& R. K. Didham (eds.). Canopy arthropods. London, Chapman \& Hall, $567 \mathrm{p}$.

Adis, J.; A. Y. Harada; C. R. V. da Fonseca; W. Paarmann \& J. A. Rafael. 1998a. Arthropods obtained from the Amazonian tree species "Cupiuba" (Goupia glabra) by repeated canopy fogging with natural pyrethrum. Acta Amazônica 28(3): 273-283.

Adis, J.; Y. Basset; A. Floren; P. M. Hammond \& K. E. Linsenmair. 1998b. Canopy fogging of an overstory tree - recommendations for standardization. Ecotropica 4: 93-97.

Adler, G. H. \& D. W. Kestell. 1998. Fates of Neotropical tree seeds influenced by spiny rats (Proechimys semispinosus). Biotropica 30(4): $677-681$

Allison, A.; A. Samuelson \& S. E. Miller. 1997. Patterns of beetle species diversity in Castanopsis acuminatissima (Fagaceae) trees studied with canopy fogging in mid-montane New Guinea rainforest, $\mathrm{p}$. 224-236. In: N. E. Stork; J. Adis \& R. K. Didham (eds.). Canopy arthropods. London, Chapman \& Hall, 567 p.

Arnett, R. H. JR. 1963. The beetles of the United States. Washington D. C., The Catholic University of America Press, xi +1112 p.

BASSET, Y. 2001. Invertebrates in the canopy of tropical forests: how much do we really know?, p.87-107. In: K. E. Linsenmair; A. J. Davies; B. Fiala \& M. R. Speight (eds.). Tropical forest canopies: ecology and management. London, Kluwer Academic Publisher, $370 \mathrm{p}$.

Basset, Y.; N. D. Springate \& G. A. Samuelson.1994. Feeding habits and range of body size: a case study in Papua New Guinea using arboreal leaf-beetles and weevils (Coleoptera: Chrysomelidae, Curculionidae) Bulletin de la Société Entomologique Suisse 67: 347-361.

Basset, Y.; V. Novotny; S. E. Miller \& R. L. Kitching. 2003. Arthropods of tropical forests. Spatio-temporal dynamics and resource use in the canopy. Cambridge, Cambridge University Press, $474 \mathrm{p}$.

Campelo JR.; A. Sandanielo; C. Caneppele; N. Priante-Filho \& C. R. De Musis. 1997. Plano de Conservação da Bacia do Alto Paraguai Relatório Técnico, p. 296-334. Vol II, Tomo I. Brasília, Ministério do Meio Ambiente e Recursos Hídricos e da Amazônia Legal.

Costa, E. C.; D. Link; G. Rosado-Neto; A. D. Grutzmacher \& J. A. S. FrançA. 1992. Entomofauna de inflorescência de palmáceas: 1 Curculionidae (Coleoptera). Anais do $9^{\circ}$ de Congresso de Entomologia 2: 949-954.

Davies, J. G.; N. E. Stork; M. J. D. Brendell \& S. J. Hine. 1997. Beetle species diversity and faunal similarity in Venezuelan rainforest tree canopies. p. 85-103. In: N. E. STORK; J. AdIs \& R. K. DidHAm (eds.). Canopy arthropods. London, Chapman \& Hall, 567 p.

Delgado, C. 2002. The relationship between Pachymerus cardo (Fahraeus) (Coleoptera: Bruchidae) and the palm Orbignya spectabilis (C. Martius) Burret (Arecaceae: Cocoeae) in a the terra firme forest, Brazilian Amazonan. Amazoniana 17(1/2): 169-172.

DidHAM, R. K. 1997. Dipteran tree-crown assemblages in a diverse southern temperate rain forest, p. 320-343. In: N. E. STORK. J. AdIs \& R. K. DiDHAm (eds.). Canopy arthropods. London, Chapman \& Hall, 567 p.

Dulmen, A. Van. 2001. Pollination and phenology of flowers in the canopy of two contrasting rain forest types in Amazonia, Colombia, p. 73-85. In: K. E. Linsenmair; A. J. Davies; B. Fiala \& M. R. Speight (eds.). Tropical forest canopies: ecology and management. London, Kluwer Academic Publisher, 370 p.

ERwin, T. L. 1983a. Tropical forest canopies: the last biotic frontier. Bulletin of the Entomological American Society 30:14-19.

ERwIN, T. L. 1983b. Beetles and other insects of tropical forest canopies at Manaus, Brazil, sampled by insecticidal fogging, p. 59-75. In: S. L. Sutton; T. C. Whitmore \& A. C. Chadwick (eds.). Tropical rain forests: ecology and management. Oxford, Blackwell Scientific Publications, $498 \mathrm{p}$.

FeEny, P. 1975. Biochemical coevolution between plants and their insect herbivores, p. 3-19. In: L. E. Gilbert \& P. Raven (eds.). Coevolution of animal and plants. Austin, University of Texas Press, $130 \mathrm{p}$. FeENy, P. 1976. Plant apparency and chemical defense. Recent Advanced 
Phytochemistry 10: 1-40.

Flechtmann, C. H. W. 1997. Mite (Arthropoda: Acari) associates of palms (Arecaceae), in Brazil: III. Eutetranychus nomurai n. $s p$ (Tetranychidae) from Attalea phalerata Mart. International Journal of Acarology 23(4): 269-273.

Floren, A. \& K. E. Linsenmair. 1997. Diversity and recolonization dynamics of selected arthropod groups on different tree species in a lowland rainforest in Sabah, Malaysia, with special reference to Formicidae, p. 344-381. In: N. E. Stork; J. Adis \& R. K. Didham (eds.). Canopy arthropods. London, Chapman \& Hall, $567 \mathrm{p}$.

Floren, A. \& K. E. Linsenmair. 1998. Diversity and recolonization of arboreal Formicidae and Coleoptera in a lowland rain forest in Sabah, Malaysia. Selbyana 19(2): 155-161.

Franco, M. S. S. \& R. Pinheiro. 1982. Geomorfologia. Levantamento de recursos naturais, Folhas SE.20 e SE.21, v. 27, p. 161-224. Projeto RADAMBRASIL. Rio de Janeiro, Ministério das Minas e Energia

Hammond, P. M. 1990. Insect abundance and diversity in the DumogaBone National Park, N. Sulawesi, with special reference to the beetle fauna of lowland rain forest in Toraut region, p. 197-254. In: W. J. Knigt \& J. D. Holloway (eds.). Insects and the rain forest of South East Asia (Wallacea). London, Royal Entomological Society of London, $343 \mathrm{p}$.

Hammond, P. M.; R. L. Kitching \& N. E. Stork. 1996. The composition and richness of the tree-crown Coleoptera assemblage in an Australian subtropical forest. Ecotropica 2: 99-108 p.

Harada, A. Y. \& J. Adis. 1997. The ant fauna of tree canopies in Central Amazonia: a first assessment, p. 382-400. In: N. E. STORK; J. ADIS \& R. K. Didham (eds.). Canopy arthropods. London, Chapman \& Hall, 567 p.

HaradA, A. Y. \& J. Adis. 1998. Ants obtained from trees of "Jacareuba" (Calophyllum brasilense) forest plantation in Central Amazonian by canopy fogging: first results. Acta Amazônica 28(3): 309-318.

Harms, K. E. \& J. W. Dalling. 2000. A bruchid beetle and a viable seedling from a single diaspore of Attalea butyracea. Journal of Tropical Ecology 16: 319-325.

Henderson, A.; G. Galeano \& R. Bernal. 1995. Field guide to the palms of the Americas. Princeton, Princeton University Press, $363 \mathrm{p}$.

HöFer, H.; A. D. Brescovit; J. AdIS \& W. PAarmann. 1994.The spider fauna of Neotropical tree canopies in Central Amazonia: First results. Studies on Neotropical Fauna and Environment 29(1): 23-32.

Hurtado Guerrero, J. C.; C. R. V. Da. Fonseca; P. M. Hammond \& N. E. STORK. 2003. Seasonal variation of canopy arthropods in Central Amazon , p. 170-175. In: Y. BAsSET; V. R. Novotny; S. E. MilleR \& R. L. Kitching (eds.). Arthropods of tropical forests. Spatiotemporal dynamics and resource use in the canopy. Cambrige, Cambridge University Press, $474 \mathrm{p}$.

JANZEN, D. H. 1971. The fate of Scheelea rostrata fruits beneath the parent tree: Predispersal attack by bruchids. Principes 15: 89-101.

JardiM, M. A. G. \& A. C. DA. C. Cunha. 1998. Usos de palmeiras em uma comunidade ribeirinha do estuário Amazônico. Boletim do Museu Paraense Emilio Goeldi, Série Botânica, 14(1): 69-77.

Johnson, C. D.; S. Zona \& J. A. NiLsson. 1995. Bruchid beetles and palm seeds: recorded relationships. Principes 39(1): 25-35.

Kahn, F. \& J-J. de Granville. 1995. Palms in forest ecosystems of Amazonia. Ecological Studies 95, Berlin, 226 p.

Kitching, R. L.; H. Mitchell; G. Morse \& G. Thebaud. 1997. Determinants of species richness in assemblages of canopy arthropods in rainforests, p.131-150.In: N. E. Stork; J. Adis \& R. K. Didham (eds.). Canopy arthropods. London, Chapman \& Hall, 567 p.

Küchmeister, H.; A. C. WebBer; I. SilberbaUer-GotTSBERGer \& G. GotTsberger, 1998. A polinização e sua relação com a termogênese em espécies de Arecaceae da Amazônia Central. Acta Amazonica 28(3): 217245.

Lawrence, J. F. \& E. B. Britton. 1991. Coleoptera, p. 543-683. In: CSIRO Division of Entomology (ed.). The insects of Australia. A textbook for students and research workers. $2^{\text {nd }}$ edition, Melbourne, University Press, 1137 p.

Lawton, J. H. 1978. Host-plant influence on insect diversity: the effects of space and time. Symposium of the Royal Entomological Society of London 9: 105-125.
Lawton, J. H. 1982. Vacant niches and insatured communities: a comparison of bracken herbivores at sites on two continents. Journal of Animal Ecology 51: 573-595.

Link, D. \& F. A. Alvarez. 1979. Palmeiras atacadas por lagartas de Brassolidae (Lepidoptera) em Santa Maria- RS. Revista do Centro de Ciências Rurais 9(2): 221-254.

Linsenmair, K. E.; A. J. Davis; B. Fiala \& M. R. Speight. 2001.Tropical forest canopies: ecology and management. Dordrecht, Kluwer Academic Publishers, $370 \mathrm{p}$.

Listabarth, C. 1999. Pollination studies of palm populations: a step toward the application of a biological species concept, vol. 83, p. 79-93. In: W. R. Buck \& W. W. Thомаs (eds.). Evolution, variation, and classification of palms. New York, The New York Botanic Garden Press, 324 p.

Macambira, M. L. J. \& M. A. G. JARDiM. 1997. Identificação dos insetos visitantes de inflorescências da palmeira inajá (Maximiliana maripa L.). Boletim do Museu Paraense Emilio Goeldi, Série Zoologia, 13(1): 85-94.

Magurran, A. E. 1988. Ecological diversity and its measurement. London, Chapman \& Hall, 179 p.

Marques, M. I.; J. Adis; C. Nunes da Cunha \& G. B. Santos. 2001. Arthropod biodiversity in the canopy of Vochysia divergens Pohl (Vochysiaceae), a forest dominant in the Brazilian Pantanal. Studies on Neotropical Fauna and Environment 36(3):205-210.

Miranda, I. P.; A. Rabelo; C. R. Bueno; E. M. Barbosa \& M. N. S. Ribeiro. 2001. Frutos de palmeiras da Amazônia, 19 ed. Manaus, Instituto de Pesquisas da Amazônia - INPA/MCT,120 p.

Mora Urpi, J. 1982. Polinización en H.B.K. (Palmae): Nota adicional. Revista de Biologia Tropical 30: 174-176.

Mora UrPi, J. \& M. Solis. 1980. Polinización en H.B.K. (Palmae). Revista de Biologia Tropical 28: 153-174.

Moran, V. C. \& T. R. E. Southwood. 1982. The guild composition of arthropod communities in trees. Journal of Animal Ecology 51: 289-306.

Nitsson, J. A. \& C. D. Johnson. 1993. A taxonomic revision of the palm bruchids beetles (Pachymerini) and a description of the world genera of Pachymerinae (Coleoptera; Bruchidae). Memoirs of the American Entomologycal Society 41: 1-104.

Novotny, V. \& Y. BASSET. 2000. Rare species in communities of tropical insect herbivores: pondering the mystery of singletons. Oikos 89: 564-572.

NunEs DA CunHA, C. \& W. J. Junk. 1999. Composição florística de capões e cordilheiras: localização das espécies lenhosas quanto ao gradiente de inundação no Pantanal de Poconé, MT-Brasil, p. 387-405. Anais do II Simpósio sobre Recursos Naturais e Sócio-Econômicos do Pantanal. Corumbá, EMBRAPA-Pantanal, 537 p.

Nunes da Cunha, C. \& W. J. JunK. 2001.Distribution of wood plant communities along the flood gradient in the Pantanal of Poconé, Mato Grosso, Brazil. International Journal of Ecology and Environment Sciences 27: 63-70.

Quiroga-Castro, V. D \& A. L. RoldÁn. 2001.The fate of Attalea phalerata (Palmae) seeds dispersed to a tapir latrine. Biotropica 33(3): 472477.

Ribeiro, S. P. \& V. K. BRown. 1999. Insect herbivory in tree crowns of Tabebuia aurea and T. ochracea (Bignoniaceae) in Brazil: contrasting the cerrado with the "Pantanal Matogrossense". Selbyana 20(1): 159-170.

Scariot, A. O.; E. Lleras \& J. Hay. 1991.Reprodutive biology of the palm Acromia aculeata in Central Brazil. Biotropica 23(1): 12-22.

ShmidA, A. \& M. R. WiLson. 1985. Biological determinants of species diversity. Journal of Biogeography 12: 1-20.

SchмidT, R. 1970. Notes on the reprodutive biology of Asterogyne martiana (Palmae). II. Pollination by syrphid flies. Principes 14: 39-49.

Southwood, T. R. E. 1961. The number of species of insect associated with various trees. Journal of Animal Ecology 30: 1-8.

Southwood, T. R. E. 1978. Ecological methods with particular reference to the study of insect population. $2^{\text {nd }}$ edition. London, Chapman \& Hall, 524 p.

Southwood, T. R. E.; V. C. Moran \& C. E. J. Kennedy. 1982. The richness, abundance and biomass of the arthropod communities on trees. 
Journal of Animal Ecology 51: 635-649.

Stork, N. E. 1988. Insect diversity: facts, fiction and speculation. Biological Journal of the Linnean Society 35:321-337.

STORK, N. E. 1991. The composition of arthropod fauna of Bornean lowland rain forest trees. Journal of Tropical Ecology 7: 161-180.

Stork, N. E. \& M. J. D. BRendell. 1990. Variation in the insect fauna of Sulawesi trees with season, altitude, and forest type, p. 173-190. In: J. W. Knigth, \& J. D. Halloway (eds.). Insects and the rain forests of South East Asia (Wallacea). London, Royal Entomologycal Society of London, $343 \mathrm{p}$.

Stork, N. E. \& M. J. D. Brendell. 1993. Arthropod abundance in lowland rain forest of Seram, p. 115-130. In: I. D. Edwards; A. A. MacDonald \& J. PвостоR (eds.). Natural history of Seram: Malukku, Indonesia. Andover, Intercept, $240 \mathrm{p}$.

Stork, N. E. \& P. M. Hammond. 1997. Sampling arthropods from treecrowns by fogging with insecticides: lessons from studies of oak tree beetle assemblages in Richmond Park (UK), p. 3-27. In: N. E. SтоRк; J. Adis \& R. K. Didham (eds.). Canopy arthropods. London, Chapman \& Hall, $567 \mathrm{p}$.

Stork, N. E.; J. Adis \& R. K. Didham. 1997. Canopy arthropods. London, Chapman \& Hall, $567 \mathrm{p}$.

TARIFA, J. R. 1986. O sistema climático do Pantanal. Da compreensão do sistema à definição de prioridades de pesquisa climatológica, p. 9-27.
Anais do I Simpósio sobre Recursos Naturais e SócioEconômicos do Pantanal. Brasília, EMBRAPA-DDT.

Tobin, J. E. 1991. A Neotropical rain forest canopy ant community: some ecological considerations, p.536-538. In: C. R. HuxLey \& D. F. CutLer (eds.). Ant-plant interactions. Oxford, Oxford University Press, $601 \mathrm{p}$.

VASCONCELOS, H. L. DE. 1990. Effects of litter collection by understory palms on the associated macroinvertebrate fauna in Central Amazonia. Pedobiologia 34: 157-160.

WAGNER, T. 1997.The beetle fauna of different tree species in forests of Rwanda and East Zaire, p. 169-183. In: N. E. StorK; J. AdIS \& R. K. Didham (eds.). Canopy arthropods. London, Chapman \& Hall, 567 $\mathrm{p}$.

Wilson, E. O. 1987. The arboreal ant fauna of Peruvian Amazonan forests: a first assessment. Biotropica 19: 245-251.

Wilson, D. E. \& D. H. JANZEN. 1972. Predation on Scheelea palms seeds by bruchid beetles: seed density and distance from the parent palm. Ecology 53: 954-959.

Wright, S. J. 1983. The dispersion of eggs by a bruchid beetle among Scheelea palm seeds and the effect of distance from the parent palm. Ecology 64: 1016-1021.

Wright, S. J. 1990. Cumulative satiation of a seed predator over the fruiting season of its host. Oikos 58: 272-276. 